\title{
Surgical models for computer-assisted neurosurgery
}

\author{
P. Jannin ${ }^{\text {a,b,c }}$, X. Morandi ${ }^{\text {a,b,c,d }}$ \\ a INSERM, U746, Faculté de Médecine CS 34317, F-35043 Rennes Cedex, France \\ ${ }^{\mathrm{b}}$ INRIA, VisAGeS Unit/Project, F-35042 Rennes, France \\ ${ }^{\mathrm{c}}$ Univ. Rennes I, CNRS, UMR 6074, IRISA, F-35042 Rennes, France \\ ${ }^{\mathrm{d}}$ Department of Neurosurgery, Pontchaillou University Hospital, Rennes, France.
}

Keywords: multimodal image-guided neurosurgery, surgical models, surgical ontology, surgical workflows, data mining, patient-specific model, computer-assisted surgery

Address for correspondence:

Pierre JANNIN

INSERM, U746, Faculté de Médecine CS 34317, F-35043 Rennes Cedex, France

Ph: +33 2232345 88; Fx: +33 29984 71 71; pierre.jannin@inria.fr 
Jannin P, Morandi X. Surgical models for computer-assisted neurosurgery. Neuroimage. 2007 Sep 1;37(3):783-91

\section{Abstract:}

In this paper, we outline a way to improve computer-assisted neurosurgery using surgical models along with patient-specific models built from multimodal images. We propose a methodological framework for surgical models that includes the definition of a surgical ontology, the development of software for describing surgical procedures based on this ontology, and the analysis of these descriptions to generate knowledge about surgical practice. Knowledge generation is illustrated by two studies. 159 patients who underwent brain tumor surgery were described from postoperative reports using the surgical ontology. First, from a sub-set of 106 surgical cases, we computed a decision tree using a prediction approach that gave probability in terms of operating room patient positioning percentages and according to tumor location within one or more lobes. Second, from the whole set of 159 surgical cases, we identified 6 clusters describing families of cases according to pathology-related parameters. Results from both studies showed possible prediction of parts of the surgical procedure from pathology-related characteristics of the patient. Surgical models enable surgical knowledge to be made explicit, facilitating the surgical decision-making process and surgical planning, and improving the human-computer interface during surgery. 
Jannin P, Morandi X. Surgical models for computer-assisted neurosurgery. Neuroimage. 2007 Sep 1;37(3):783-91

\section{Introduction}

Much has been accomplished to date in the construction of numerical patient-specific models from multimodal preoperative images for the purpose of image-guided surgery. With image segmentation and registration methods, it is possible to define surgical target(s), reference areas, areas to be avoided, and trajectories based on these preoperative multimodal images [Jannin 2000]. Preoperative patientspecific models, used in surgical planning, can be displayed in the operating room (OR) with the patient, with the help of augmented reality, and updated via intraoperative imaging (e.g., MRI, 2D/3D US, video). It has been shown that these systems allow for better patient outcomes while resulting in lower morbidity and mortality. For instance, in neurosurgery, small lesions can be operated earlier, even if located in high-risk functional areas [Jannin 2002]. However, some limitations have been identified [Lemke 2005, Peters 2006]. There remains an important gap between the surgical reality its complexity and its high interpatient and intersurgeon variability - and the limited understanding of surgery currently implemented in Computer-Assisted Surgery (CAS) systems. These systems rely on little explicit, formal knowledge of surgery, or none at all. Ergonomical aspects are poorly suited to the surgical environment, which is both critical and specific. Dataflows and workflows in the surgical process are seldom correctly managed. Finally, systems provide no access to the surgical state of the art, or to current practice. These limitations are tied to high costs, process redundancies, and even some clinical failures.

For our purposes, we will clarify some concepts by proposing definitions, as required. A “surgical workflow” is defined as the automation of a business process in the surgical management of patients, in whole or part, during which documents, information, images or tasks are passed from one participant to another for action, according to a set of procedural rules (adapted from http://www.wfmc.org). "Surgical models” are related to generic or patient specific surgical procedures that surgical workflows aim to automate. As explained in more details in section 2.1, these surgical models usually include descriptions of the actual surgical procedure, i.e., activities performed by the surgeon and surgical staff as well as systems, such as imaging devices or computers. The surgical 
Jannin P, Morandi X. Surgical models for computer-assisted neurosurgery. Neuroimage. 2007 Sep 1;37(3):783-91

models are masters justifying the needs for a workflow management system. Issues regarding generic and patient-specific surgical models relate to their definition, computation or estimation.

There is a strong need for making explicit surgical know-how and associated scenarios in computer assisted surgical systems through surgical models. It has been previously discussed with regard to surgical simulation [Satava 1996, Taylor 1999], robotics systems [Munchenberg 2000] and minimally invasive surgery [MacKenzie 2001]. Until very recently, there have been few concrete initiatives. We can divide them into 3 categories based on their objectives: reporting-centered approaches, operative approaches, and approaches targeting perioperative workflow optimization. The reporting-centered approaches have focused on nomenclature generation and translation [CEN 2001, Price 1998, Trombert-Paviot 2000]. The operative approaches focused on optimizing human/device interfaces by modeling interactive aspects [Kragic 2003, Mansoux 2005, Trevisan 2003], on studying the surgical gesture to directly optimize surgical planning [MacKenzie 2001, Munchenberg 2000], or on studying surgeons’ gestures for partial robotic assistance [Botturi 2005, Kragic 2003, Nageotte 2005]. Finally, the need for perioperative surgical workflow optimization has recently emerged, especially regarding the specifications of the operating room of the future [Dickhaus 2004, Fischer 2005, Lemke 2004, Lemke 2005, Sandberg 2004]. In most of these publications, the methodology employed to build surgical models was not explicitly described. For operative approaches as well as approaches targeting workflow optimization, no ontology was used, making it impossible to use outcomes in other surgical specialties or share knowledge between systems and research groups. Finally, few of the approaches were implemented, making it difficult to grasp their potential added value and their relevance. Our hypothesis is that surgical models are needed for improving computer aided surgery, and that there exist invariant surgical models that can be made explicit via careful retrospective analysis of surgical data and information.

In this paper, we introduce a methodological framework for surgical procedures modeling and present the results of an initial implementation of this framework in brain surgery. We present the prospective 
Jannin P, Morandi X. Surgical models for computer-assisted neurosurgery. Neuroimage. 2007 Sep 1;37(3):783-91

use of surgical models for surgical planning of 19 patients and two studies showing possible prediction of parts of the surgical procedure from pathology-related characteristics of the patient.

\section{Materials and methods}

We propose the following methodology for modeling surgical procedures. The design of this methodology was derived from knowledge modeling and cognitive systems engineering [Rasmussen 1994]. It consists of the following steps:

1. Definition of the modeling objective (i.e., aim of modeling approach);

2. Definition of the surgical work domain to be modeled (i.e., universe of discourse);

3. Definition of an ontology for the work domain which involves identification of concepts and relationships describing the surgical work domain, choice of a formalism for representing concepts and relationships, implementation of the formalized ontology into prototype software, and test of the prototype for testing previous steps;

4. Data Acquisition which consists in describing surgical cases using the formalized ontology;

5. Visualization and browsing of descriptions;

6. Analysis by knowledge extraction from these descriptions which involves distinction between predicted and predictive parameters, data pre-processing, and data mining;

7. Evaluation of generated knowledge.

Steps from 1 to 6 have been implemented in our clinical environment. Steps 1 to 3 were introduced in a previous publication [Jannin 2003] within the context of image-guided neurosurgery and are briefly presented in this paper. Steps 4 to 6 are introduced for knowledge generation in brain surgery.

\subsection{Definition of modeling objective, work domain, and ontology}

Clear and accurate definition of modeling objectives and work domains is a crucial initial step in cognitive systems engineering [Rasmussen 1994] and therefore also in the proposed methodology. The surgical process may be tackled from different perspectives according to the surgical timeline: perioperative, preoperative, intraoperative, or postoperative perspectives. Each of these aspects may be studied at different levels of granularity. Each study may focus on actors (i.e., humans or devices), 
Jannin P, Morandi X. Surgical models for computer-assisted neurosurgery. Neuroimage. 2007 Sep 1;37(3):783-91

tasks, systems, states or on the information or knowledge involved. Static or dynamic aspects may also be studied. In this paper, the work domain we model is related to the mental image the surgeon has about the surgical procedure performed in the OR, for one specific patient. This mental image, which includes the main surgical steps and their sequencing, was captured through interviews with surgeons and studies of postoperative surgical reports. Our modeling was aimed at making this mental image explicit along with part of the knowledge used by surgeons to build it. We restricted our domain of interest to surgeries for brain tumors and brain cavernomas.

Building an ontology is a crucial and required step in modeling a work domain [Chandrasekaran 1999]. It provides a language for describing objects and relationships within this domain. We built the ontology of our work domain, described above, by identifying the main concepts, relationships, and cardinalities of our work domain from descriptions of surgical cases by neurosurgeons. We formalized these main concepts and relationships in a UML class diagram (Unified Modeling Language) (Fig. 1). This work was presented in [Jannin 2003].

\subsection{Implementation of the ontology into surgical planning software} We implemented the formalized ontology inside a surgical planning software (Fig. 2) allowing the number of surgical steps to be defined for each surgical procedure. For each step, the surgeon was able to assign the corresponding action and the relevant image entities selected from a list of 3D images and surfaces segmented from multimodal preoperative images of the patient. The surgeon assigned a role and visualization parameters (e.g., color, transparency value) to each image entity. Each procedure was stored in a structured form as an XML (Extensible Markup Language) file. For each step, a 3D scene including all 3D image entities and visualization parameters was stored as an XML file and as a VTK (Visualization ToolKit) file. A second implementation consisted of web-based software developed with the $P h P$ language. Descriptions were stored in a relational database (PostGreSQL) and in XML files. Both implementations and use of them allowed validation and refinement of our model. 
Jannin P, Morandi X. Surgical models for computer-assisted neurosurgery. Neuroimage. 2007 Sep 1;37(3):783-91

\subsection{Data acquisition and visualization}

From a global population of 189 patients who underwent brain surgery, we distinguished two groups. The first group included 19 surgical cases (15 men, 36.5 years \pm 12.7 ; 4 women, 36.5 years \pm 10.6 ) with lesions located in eloquent areas (8 surgeries for brain tumors and 11 surgeries for brain cavernomas). All of them underwent multimodal neuronavigation as described elsewhere [Jannin 2002] and were prospectively described using the UML class diagram, with the planning software before surgery. For these 19 surgical cases, the planning software was used pre and intra operatively to display the different surgical steps along with the corresponding 3D scenes (Fig. 2). The second group included 170 additional surgical cases with brain tumors (113 men, 48.2 years \pm 15.7 ; 57 women, 54.7 years \pm 13.7 ). All of them were retrospectively described by 4 senior neurosurgeons ( 2 full professors and 2 associate professors with about 25 and 15 years expertise in surgical practice, respectively) from postoperative reports, using the web-based software, and stored both in a SQL database and as XML files. From this second group we excluded 11 rare cases, such as left-handed patients (3), patients with tumors in both hemispheres (2), patients with trans-sulcal approach (1), patients with two successive trans-gyral approaches (2) and patients with more than a single pathological target (3). Finally, these 159 surgical descriptions consisted in 103 men (49.9 years \pm 15.5$)$ and 56 women (54.8 years \pm 13.7$)$. Data visualization allowed displaying descriptions of surgical cases based on simple requests using criteria based on values of the ontology's concepts [Raimbault 2005]. Available criteria included name of the pathology, its location in a lobe or gyrus, and surgical department. Results of the request were displayed as a list of corresponding surgical cases. For each surgical case, the surgeon was able to see the description of the whole surgical procedure as a list of surgical steps and related information.

\subsection{Data analysis: Extracting surgical expertise from descriptions}

Data analysis was aimed at explicitly understanding the work domain through its descriptions. We oriented the analysis toward the study of invariance between surgical cases. We assumed that there was invariance within subsets of surgical cases and that the distinction between these subsets depended on patient-related parameters. We attempted to extract this invariance from a surgical 
Jannin P, Morandi X. Surgical models for computer-assisted neurosurgery. Neuroimage. 2007 Sep 1;37(3):783-91

database in order to predict aspects of the surgical procedure based on patient-related parameters. The methodology we used included the following steps: (a) distinguishing between predicted and predictive parameters, (b) data pre-processing, and (c) data mining. This methodology was used for two studies.

Distinguishing between predicted and predictive parameters: Surgical cases included in the second group were described using the ontology (i.e. by classes from the model shown in Fig. 1) from postoperative reports. Each class includes one or more parameters. We distinguished predictive parameters from predicted parameters as follows. Predictive parameters belong to classes describing the surgical case (Fig. 3a). These classes include patient information, surgery type, and name of the pathology, its depth, and its hemisphere, lobe and gyrus of location. This information is known before surgery. Predicted parameters belong to classes describing surgical procedure (Fig. 3b). These classes include information about the different surgical steps, the corresponding actions, action attributes such as patient positioning value and shape of the skin incision, action models, and the anatomical, pathological, and functional structures the surgeon considered relevant to performing this specific action. The objective of data analysis was to predict the course of surgery (described by the predicted parameters) from the description of the surgical case (provided by the predictive parameters).

Data preprocessing: The 159 surgical descriptions from the second group were translated into the flat representations required for data mining approaches.

Data mining: We first studied the relationship between patient positioning in the OR and the anatomical location of tumors in the sub-group of 159 patients. In this study, we used a prediction approach involving computation of decision trees using Cart software (Salford Systems, San Diego, CA, USA). We randomly divided the sub-group of 159 surgical cases into a learning set of 106 surgical cases and a testing set of 53 surgical cases. The testing set allowed evaluating prediction error. Possible predictive values for tumor location involved the following 4 lobes: temporal (42 surgical cases from 159), parietal (24 cases), occipital (5 cases), and frontal (59 cases) lobes. 4 surgical cases 
Jannin P, Morandi X. Surgical models for computer-assisted neurosurgery. Neuroimage. 2007 Sep 1;37(3):783-91

had tumor located in both frontal and parietal lobes, 6 in both frontal and temporal lobes, 2 in both parietal and occipital lobes, one in both occipital and temporal lobes, 15 in both parietal and temporal lobes, and one in frontal, parietal and temporal lobes. Possible predicted values for patient positioning involved the following 3 positions: dorsal (i.e., supine), ventral (i.e., prone), or lateral decubitus. With this approach, we computed a decision tree, decision rules, and a confusion matrix for evaluating classification errors.

The second study focused on another methodological approach for data mining. We wanted to identify clusters of surgical procedures, assuming that there were relationships between families of patients with similar characteristics and families of surgical procedures with similar characteristics. Therefore, we classified our sub-group of 159 surgical cases based on the predictive parameters describing pathology-related parameters by using a K-means approach, followed by an ascending hierarchical clustering. K-means identified number of clusters and gave a dendogram as a result. From the dendogram, we decided to distinguish 6 clusters. Ascending hierarchical clustering classified each surgical case into one of these 6 clusters. We then manually studied the predicted parameters values within each cluster in order to identify invariant parameters in surgical procedures.

\section{Results}

The ontology's main concepts and relationships were identified and formalized in a UML class diagram (Fig. 1). The major concepts included in the ontology follow. The surgical procedure is broken down into a sequential list of surgical steps. Each step is described by an action (such as positioning or incision) with associated attributes (such as patient position or shape of skin incision) and includes a list of anatomical, pathological or functional structures which the action affects (such as patient's body for patient positioning or skin for skin incision). Each structure may be represented by an image entity extracted from multimodal images of the patient (such as skin segmented from MR images). The role of each image entity is specified: target area (such as tumors or malformations), area to be avoided (such as high-risk functional areas or vessels), reference area (such as cortical sulci or 
Jannin P, Morandi X. Surgical models for computer-assisted neurosurgery. Neuroimage. 2007 Sep 1;37(3):783-91

vessels), or surgical approach. More explanation is available in [Jannin 2003].

In the first data analysis study, we computed the decision tree using a prediction approach giving the probability of patient positioning as a percentage of the learning set and according to tumor location inside one or more lobes (Fig. 4). The classification error was $18 \%$ when computed on the learning set and $20 \%$ when computed on the testing set. Results showed the relationship between tumor location inside a lobe and patient positioning in the OR. Classification errors emphasized the need for a finer description of both tumor location and patient positioning.

In the second data analysis study, from the sub-group of 159 surgical cases with brain tumor we automatically identified 6 clusters describing families of surgical cases according to pathology-related parameters (Table 1). The number of cases per cluster is given in the black rows. Then, for each cluster, the first rows give information about values of pathology-related parameters and the grey rows give certain parameters related to the surgical procedure and considered relevant for the cluster (i.e., with higher percentages than for the whole set). The second column gives the percentage of surgical cases from the cluster verifying the corresponding property; the third column gives the percentage of surgical cases from the whole set verifying the corresponding property. The results showed a possible classification of surgical procedures from pathology-related parameters, and, for each cluster, possible predicted values, such as patient positioning, surgical approach and number of surgical steps. For example, for cluster 5 consisted of a set of 25 surgical cases with regular benign tumor, analysis shown that 22 cases were approached in a different way than transgyral approach.

\section{Discussion}

The major goal of the approach presented was to make aspects of surgical knowledge involved in the surgical process explicit. This knowledge usually comes from two different sources: learning from experts or learning from one's own surgical experience. Learning from one's own experience provides additional information about standard procedures, and especially about surgical procedures with no or low accepted consensus and high variability. Acquiring surgical knowledge from daily practice (i.e., from surgical cases) reduces risk of subjective opinion, missing important specific surgical cases, and 
Jannin P, Morandi X. Surgical models for computer-assisted neurosurgery. Neuroimage. 2007 Sep 1;37(3):783-91

perfectly addresses the high interpatient variability, since each surgical case is taken into account during knowledge generation according to its statistical representativity. On the other hand, gathering surgical cases from different surgeons allows the intersurgeon variability to be considered.

In this paper, the ontology served as a basis for describing surgical cases and ensured description accuracy relative to the targeted work domain. Building an ontology is a challenging task. It is difficult to ensure that the work domain is fully represented in the ontology, at least relative to the modeling objective. Using an upper-level ontology as a basis for defining the generic concepts of our ontology could be a means of ensuring consistency with other ontologies built on the same upper-level ontology. One limitation of our ontology is that it was built from a limited number of surgical cases and with one surgical department involving only 4 neurosurgeons. Consequently, the resulting ontology may only reflect local surgical practices. Additionally, different facets of the surgical work domain can be studied and modeled. For instance, in [Neumuth 2006, Siddoway 2006], the physical actions performed by human operators including surgeons and surgical staff are captured using a dedicated ontology. There is a clear need to define a global framework for surgical modeling based on upper-level ontologies [Burgert 2006] for creating coherence between models of different facets of surgery.

The planning software was used to describe 19 surgical cases before surgery, including all surgical steps. No quantitative assessment was performed, but the neurosurgeons found that identifying major surgical steps with the relevant images during planning was useful for patient-based surgical rehearsal. It helped the surgeons anticipate key surgical steps, certain operative views of the patient and his/her images, and key structures to be identified during surgery. The software was also used to display, in the OR, the different surgical steps of the procedure as defined during planning, along with the corresponding 3D images. Neurosurgeons also found it useful that, during surgery, for the performance of each surgical step identified during planning, only the selected relevant images were displayed. The impact of both features must be quantitatively evaluated. This surgical model-based 
Jannin P, Morandi X. Surgical models for computer-assisted neurosurgery. Neuroimage. 2007 Sep 1;37(3):783-91

approach is a new concept in image-guided surgery, both for surgical planning and intervention. It allows better anticipation of surgery by a complete planning stage encompassing all surgical steps. Moreover, user interface for Image Guided Surgical systems may be adapted according to the current surgical step as defined during planning. For instance, acting as a supervision system, it allows better management of 3D multimodal images in augmented reality systems available in the OR, by displaying relevant images when required only.

Being able to query the database of surgical cases using patient-specific parameters allowed surgeons to access descriptions of surgical procedures performed in similar cases. The corresponding surgical steps, events, surgical tools, and preoperative images can be displayed. In an initial version of the software, only parameters related to the patient's pathology were used to browse surgical cases. More patient parameters have now been implemented within more complex, combined requests.

Data mining is an interesting approach for surgical knowledge extraction. Results of the first study are quite straightforward, but they are automatically generated from an initial hypothesis. Patient positioning is of prime importance for safe and efficient conduct in cranial surgery [Hernesniemi 2005, Mohsenipour 1994, Perneczky 1999, Shevach 1992]. A comfortable working angle should be ensured by careful positioning of the patient's head and body. Furthermore, the patient should be positioned to minimize the effect of gravity, which is one of the major components of brain shift leading to loss of spatial registration with preoperative images during neuronavigation procedures [Roberts 1998]. In our first study, the choice of the position was mainly dictated according to the tumour location. Some results of the second study shown that no transgyral approach was performed for cluster 5. Indeed, when dealing with benign cerebral tumors, the approach via the subarachnoid spaces within cerebral sulci (transsulcal approach) makes them accessible while minimizing injury to surrounding normal brain tissue, as the cortex is thinner in the depth of the sulci [Harkey 1989, Yasargil 1988]. 
Jannin P, Morandi X. Surgical models for computer-assisted neurosurgery. Neuroimage. 2007 Sep 1;37(3):783-91

Both studies used different data mining methods and addressed two different issues: predicting parameters related to the surgical procedure from those related to the patient and his/her pathology, and identifying parameters underpinning the clusters of surgical procedures. Both approaches can be applied for studying other aspects of surgery. The limitations of both studies were based on the small number of samples (i.e., surgical cases) and neurosurgeons involved in these studies (i.e., surgical procedures came from only 4 different neurosurgeons). The limited number of samples can be explained by the time required to describe surgical procedures. Descriptions were made by the neurosurgeons themselves. This time-consuming task is not compatible with the overall clinical workload and needs to be automated. For the first study concerning patient positioning in brain tumor surgery, localization of tumor should be evaluated at a gyrus level for providing more statistically significant results. A number of at least 25 surgical cases per gyrus should be suitable for such analysis. Finally, we did not assess the knowledge generated for this paper, but this step is crucial prior to any knowledge diffusion.

\section{Conclusion}

In this paper, we propose a general methodology for surgical modeling. It was used in the context of neurosurgery and would have to be adapted to other surgical specialties. We focused on one specific aspect of the surgical work domain. As pointed out in this paper, other aspects of surgery are of interest and should also be studied. However, for both types of expansion (i.e., into different surgical specialties or work domains), the general methodology would remain the same. We showed how modeling patient-specific surgical procedures could impact different stages of the surgical process: choosing a surgical strategy, for patient-specific surgical rehearsal and planning, during surgery, to improve the human/computer interfaces of computer-assisted surgical systems, and finally, to formalize surgical knowledge and practice. Being able to access to explicit descriptions of surgical procedures through surgical models will also be a valuable tool for simulation software. Additionally, surgical models are a basis for surgical workflow optimization which aims at increasing quality of care and decreasing costs. It is our hope that this approach will facilitate evidence-based medicine in 
Jannin P, Morandi X. Surgical models for computer-assisted neurosurgery. Neuroimage. 2007 Sep 1;37(3):783-91

surgery by continuously updating and improving surgical knowledge based on daily surgical experience. Future works in this area include automatic acquisition of patient-specific models, development of robust and adapted methods for data mining and surgical prediction, and moreover, implementation in CAS systems and wide-scale deployment. 
Jannin P, Morandi X. Surgical models for computer-assisted neurosurgery. Neuroimage. 2007 Sep 1;37(3):783-91

\section{References}

Botturi, D., Fiorini, P., Fracastoro, G., 2005. Task level model for surgical environment. In: Troccaz, J., Merloz, P. (Eds), Proceedings of Surgetica, Chambery, France. Sauramps Médical, Montpellier, pp. 371-378.

Burgert, O., Neumuth, T., Lempp, F., Mudunuri, R., Meixensberger, J., Strauß, G., et al., 2006. Linking top-level ontologies and surgical workflows. Int J Comp Assist Radiol Surg 1 (suppl. 1), 437438.

CEN, 2001. European Committee for Standardisation prEN 1828. Structure for classification and coding of surgical procedures. Brussels CEN, September 2001.

Chandrasekaran, B., Josephson, J.R., Benjamins, V.R., 1999. What are ontologies and why do we need them? IEEE Intelligent Systems 14, 20-26.

Dickhaus, C.F., Burghart, C., Tempany, C., D'Amico, A., Haker, S., Kikinis, R., Woern, H., 2004. Workflow modeling and analysis of computer guided prostate brachytherapy under MR imaging control. Studies in Health Technology and Informatics 2004; 98:72-74.

Fischer, M., Strauss, G., Burgert, O., Dietz, A., Trantakis, C., Meixensberger, J. Lemke, H.UH, 2005. ENT-surgical workflow as an instrument to assess the efficiency of technological developments in medicine, Computer Assisted Radiology and Surgery (CARS) 2005, Berlin, Germany, Elsevier B. V., Amsterdam, pp. 1281:851-855.

Harkey H.L., al-Mefty O., Haines D.E., Smith R.R. , 1989. The surgical anatomy of the cerebral sulci. Neurosurgery 24, 651-654.

Hernesniemi J., Niemelä M., Karatas A., Kivipelto L., Ishii K., Rinne J., et al, 2005. Some collected principles of microneurosurgery: simple and fast, while preserving normal anatomy. A review. Surg Neurol 64, 195-200.

Jannin, P., Morandi, X., Fleig, O.J., Le Rumeur, E., Toulouse, P., Gibaud, B., et al., 2002. Integration of sulcal and functional information for multimodal neuronavigation. J Neurosurg 96, 713-723. 
Jannin P, Morandi X. Surgical models for computer-assisted neurosurgery. Neuroimage. 2007 Sep 1;37(3):783-91

Jannin, P., Raimbault, M., Morandi, X., Riffaud, L., Gibaud, B., 2003. Modelling surgical procedures for multimodal image-guided neurosurgery. Comp Aid Surg 8, 98-106.

Jannin, P., Fleig, O.J., Seigneuret, E., Grova, C., Morandi, X., Scarabin, J.M., 2000. A data fusion environment for multimodal and multi-informational neuro-navigation. Comp Aid Surg 5, 11-17. Kragic, D., Hager, G.D., 2003. Task modelling and specification for modular sensory based humanmachine cooperative systems. IEEE/RSJ International Conference on Intelligent Robots and Systems. IROS'03, Las Vegas, pp. 3192- 3197.

Lemke, H. U., Trantakis, C., Köchy, K., Müller, A., Strauss, G., Meixensberger, J., 2004. Workflow analysis for mechatronic and imaging assistance in head surgery. In: Lemke, H.U., Vannier, M.W., Inamura, K. et al. (Eds), Computer Assisted Radiology and Surgery. Elsevier, pp 830-835.

Lemke, H.U., Ratib, O.M., Horii, S.C., 2005. Workflow in the operating room: review of Arrowhead 2004 seminar on imaging and informatics. In: Ratib, O.M., Horii, S.C. (Eds), SPIE Medical Imaging, PACS and Imaging Informatics, Volume 5748. SPIE Press, Bellingham, pp. 5748-12.

MacKenzie, C.L., Ibbotson, J.A., Cao, C.G.L., Lomax, A.J., 2001. Hierarchical decomposition of laparoscopic surgery: a human factors approach to investigating the operating room environment. Min Invas Ther and Allied Technol 3, 121-127.

Mansoux, B., Nigay, L., Troccaz, J., 2005. Interaction between a surgeon and a computer assisted surgery system: an interactive design space. In: Troccaz, J., Merloz, P. (Eds), Proceedings of Surgetica, Chambery, France. Sauramps Médical, Montpellier, pp. 379-385.

Mohsenipour I., Goldhahn W.E., Fischer J., Platzer W., Pomaroli A. (Eds), 1994. Approaches in neurosurgery. Central and peripheral nervous system. Thieme Medical Publishers, Inc. New York. Munchenberg, J., Worn, H., Brief, J., Hassfeld, S., Muhling, J., 2000. A Pattern catalogue of surgical interventions for computer-supported operation planning. In: Westwood, J.D., Hoffman, H.M., Mogel, G.T., Stredney, D. (Eds), Medicine Meets Virtual Reality. IOS Press, Amsterdam, pp. 227- 229. 
Jannin P, Morandi X. Surgical models for computer-assisted neurosurgery. Neuroimage. 2007 Sep 1;37(3):783-91

Nageotte, F., Zanne, P., de Mathelin, M., Doignon, C., Soler, L., Leroy, J., et al., 2005. Needle path planning for suturing in laparoscopic surgery. In: Troccaz, J., Merloz, P. (Eds), Proceedings of Surgetica, Chambery, France. Sauramps Médical, Montpellier, pp. 387-395.

Neumuth, T., Durstewitz, N., Fischer, M., Strauß, G., Dietz, A., Meixensberger, J., et al., 2006. Structured recording of intraoperative surgical workflow. In: Ratib, O.M., Horii, S.C. (Eds), SPIE Medical Imaging, PACS and Imaging Informatics, Volume 6145. SPIE Press, Bellingham, pp. 614510.

Perneczky A., Müller-Forell W., van Lindert E., Fries G., (Eds), 1999. Keyhole concept in neurosurgery with endoscope-assisted microsurgery and cases studies. Thieme, Stuttgart. New York. Peters, T.M., 2006. Image-guidance for surgical procedures. Phys Med Biol 51, R505-R540. Price, C., O'Neil, M., Bentley, T.E., Brown, P.J.B., 1998. Exploring the ontology of surgical procedures in the Read Thesaurus. Methods Inf Med 35, 420-425.

Raimbault, M., Morandi, X., Jannin, P., 2005. Towards models of surgical procedures: analyzing a database of neurosurgical cases. In: Ratib, O.M., Horii, S.C. (Eds), SPIE Medical Imaging, PACS and Imaging Informatics, Volume 5748. SPIE Press, Bellingham, pp. 97-104.

Rasmussen, J., Pejtersen, A.M., Goodstein, L.P., 1994. Cognitive systems engineering. John Wiley \& Sons.

Roberts D.W., Hartov A., Kennedy F.E., Miga M.I., Paulsen K.D., 1998. Intraoperative brain shift and deformation: a quantitative analysis of cortical displacement in 28 cases. Neurosurgery 43, 749-760. Sandberg, W.S., 2004. Redesigning perioperative care for a high velocity OR. UCLA Seminar on Imaging and Informatics. http://www.radnet.ucla.edu/Arrowhead2004/

Sandberg, W.S., Daily, B., Egan, M., Stahl, J.E., Goldman, J.M., Wiklund, R.A., et al., 2005.

Deliberate perioperative systems design improves operating room throughput. Anesthesiology 103, 406-418.

Satava, R.M., Carrico, C.J., 1996. Advanced simulation technologies for surgical education. Bull Am Coll Surg 81, 71-77. 
Jannin P, Morandi X. Surgical models for computer-assisted neurosurgery. Neuroimage. 2007 Sep 1;37(3):783-91

Shevach, I., Cohen, M., Rappaport, ZH., 1992. Patient positioning for the operative approach to midline intracerebral lesions: technical note. Neurosurgery 31, 154-155.

Siddoway, D., Inghol, M.L., Burgert, O., Neumuth, T., Watson, V., Cleary, K., 2006. Workflow in Interventional Radiology: Nerve Blocks and Facet Blocks. In: Ratib, O.M., Horii, S.C. (Eds), SPIE Medical Imaging, PACS and Imaging Informatics, Volume 6145. SPIE Press, Bellingham, pp. 1-8. Taylor, C.A., Draney, M.T., Ku, J.P., Parker, D., Steele, B.N., Wang, K., et al., 1999. Predictive medicine: computational techniques in therapeutic decision making. Comp Aid Surg 4, 231-247. Trevisan, D.G., Macq, B., Vanderdonckt, J., Raftopoulos, C., 2003. Modelling interaction for imageguided procedures. In: Hanson, K., Chen, C.T., Siegel, E.L. (Eds), SPIE Medical Imaging, Volume 5029. SPIE Press, Bellingham, pp.108-118.Trombert-Paviot, B., Rodrigues, J. M., Rogers, J. E., Baud, R., van der Haring, E., Rassinoux, A. M., et al., 2000. GALEN: a third generation terminology tool to support a multipurpose national coding system for surgical procedures. Int J Med Inform 58-59, 71-85. Yasargil M.G., Cravens G.F., Roth P., 1988. Surgical approaches to “inaccessible” brain tumors. Clin Neurosurg 34, 42-110. 
Jannin P, Morandi X. Surgical models for computer-assisted neurosurgery. Neuroimage. 2007 Sep 1;37(3):783-91

Figure legends:

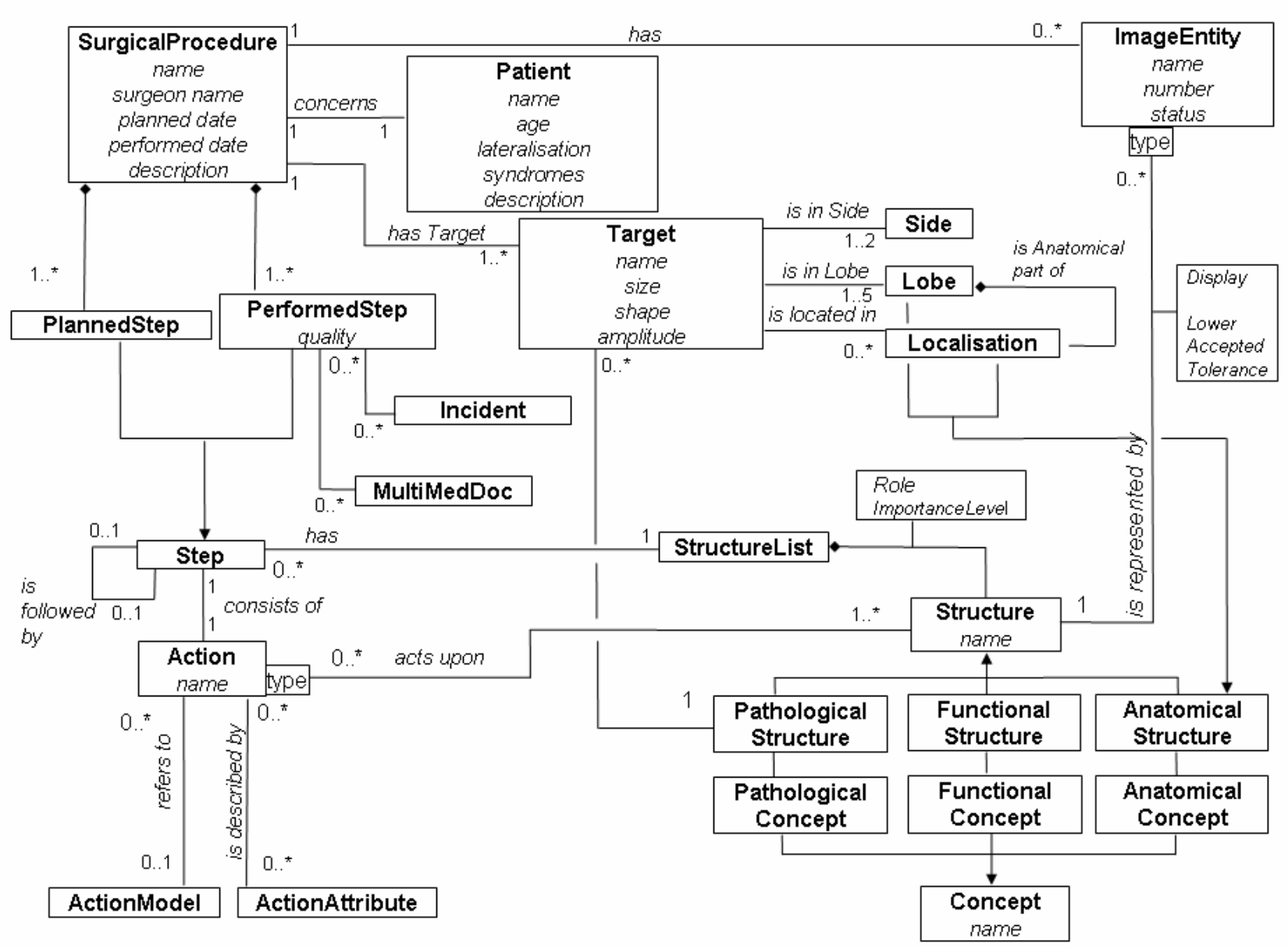

Figure 1: UML class diagram representing the surgical ontology of the surgical work domain under study. 
Jannin P, Morandi X. Surgical models for computer-assisted neurosurgery. Neuroimage. 2007 Sep 1;37(3):783-91

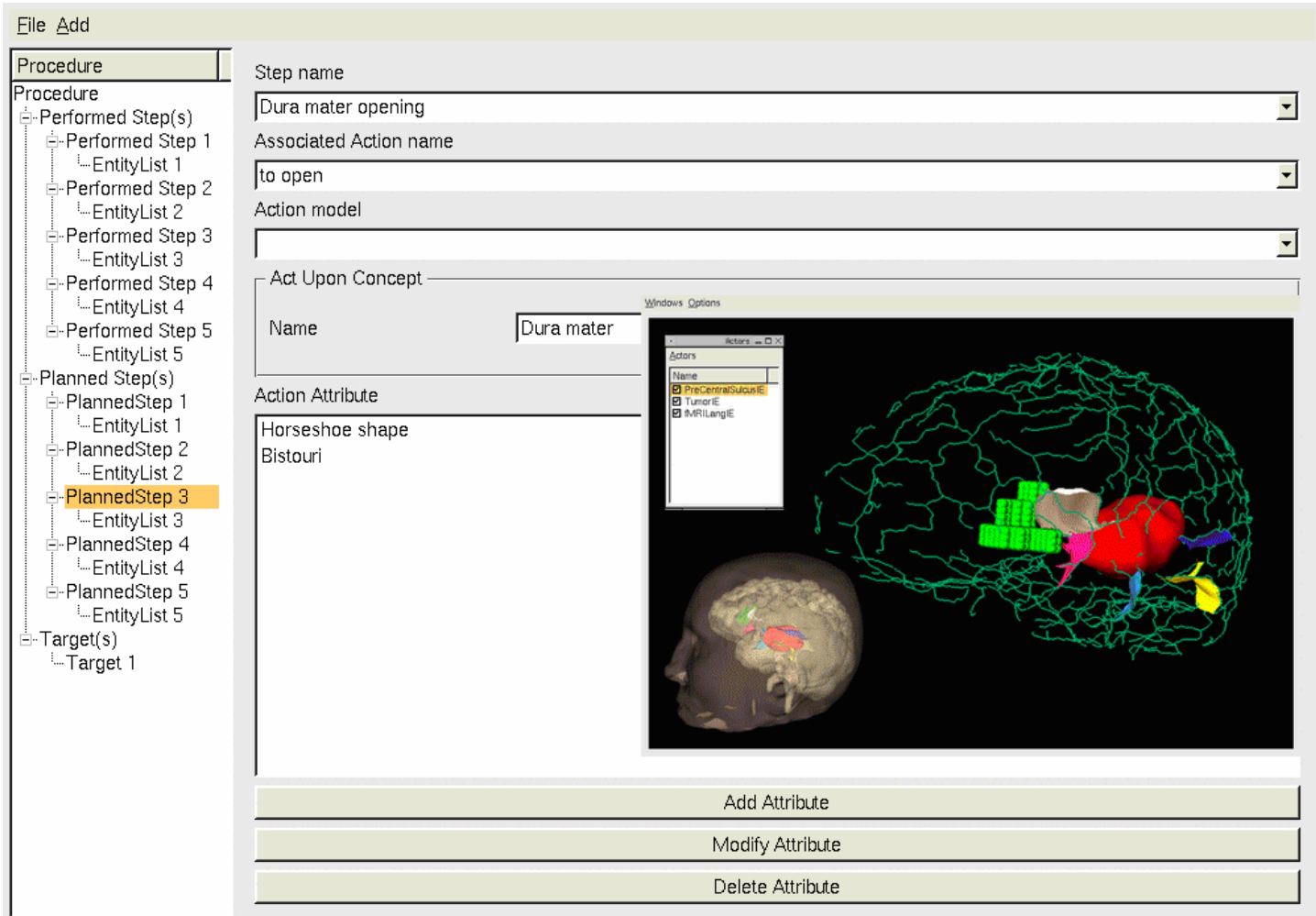

Figure 2: User interface for the planning software. Description of a surgical step and corresponding 3D scene with relevant image entities.

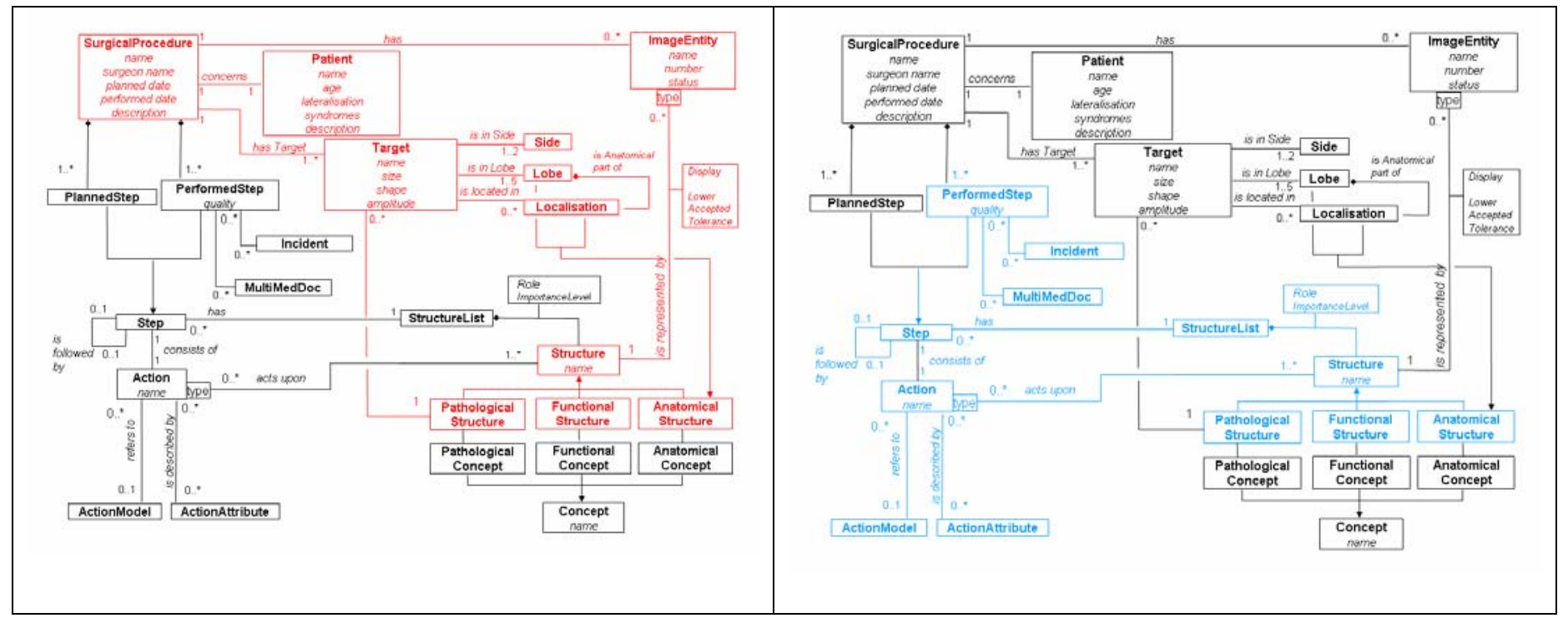

Figure 3: a) In red, predictive parameters corresponding to classes and associated relationships describing the surgical case, b) in blue, predicted parameters corresponding to classes and associated relationships describing the surgical procedure. 
Jannin P, Morandi X. Surgical models for computer-assisted neurosurgery. Neuroimage. 2007 Sep 1;37(3):783-91

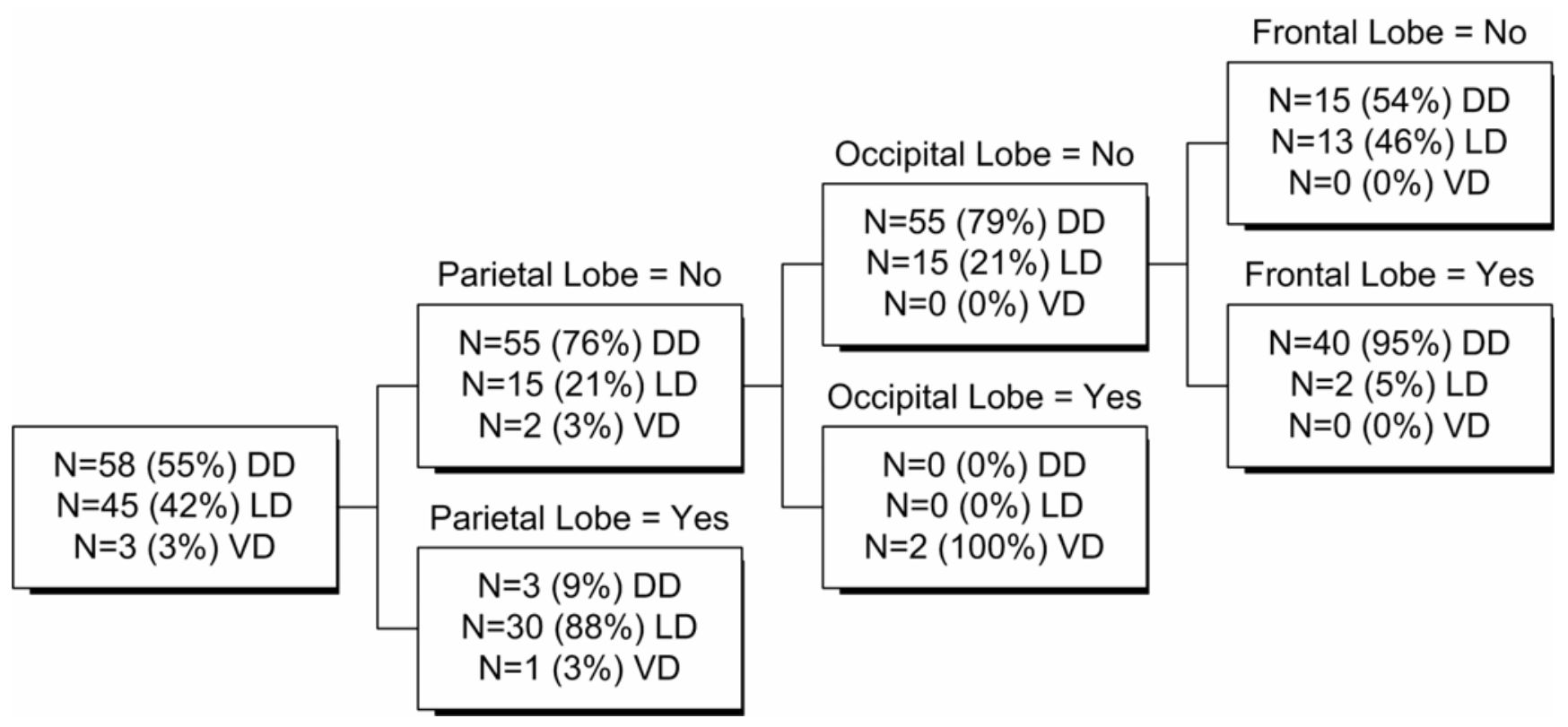

Figure 4: Decision tree for patient positioning according to tumor location in one or more cortical lobes for a population of 106 patients undergoing brain tumor surgery. DD: Dorsal Decubitus, LD: Lateral Decubitus, VD: Ventral Decubitus. 
Jannin P, Morandi X. Surgical models for computer-assisted neurosurgery. Neuroimage. 2007 Sep 1;37(3):783-91

\begin{tabular}{|c|c|c|}
\hline $\begin{array}{l}\text { Property: } \\
\text { Parameter = Value }\end{array}$ & $\begin{array}{c}\% \text { of cases from this cluster } \\
\text { verifying this property }\end{array}$ & $\begin{array}{l}\text { \% of cases from the whole } \\
\text { set verifying this property }\end{array}$ \\
\hline \multicolumn{3}{|c|}{ Cluster 1 - Number of surgical cases: 34} \\
\hline LobeTemporal = Yes & 100 & 40.88 \\
\hline LobeParietal $=$ No & 100 & 71.07 \\
\hline LobeFrontal $=$ No & 94.12 & 55.97 \\
\hline TargetRef = Malignant tumor & 97.06 & 76.73 \\
\hline TargetAmplitude $=$ Subcortical & 76.47 & 49.69 \\
\hline SkinIncisionForm =question-mark shape & 47.06 & 13.21 \\
\hline \multicolumn{3}{|c|}{ Cluster 2 - Number of surgical cases: 37} \\
\hline LobeParietal $=$ No & 100 & 28.93 \\
\hline TargetRef = Malignant tumor & 94.59 & 76.73 \\
\hline LobeFrontal $=$ No & 86.49 & 55.97 \\
\hline SkinIncisionForm = Horseshoe shape & 91.89 & 41.51 \\
\hline \multicolumn{3}{|c|}{ Cluster 3 - Number of surgical cases: 8} \\
\hline LobeOccipital = Yes & 100 & 5.03 \\
\hline LobeFrontal $=$ No & 100 & 55.97 \\
\hline PatientPosition $=$ Ventral decubitus & 75 & 4.40 \\
\hline \multicolumn{3}{|c|}{ Cluster 4 - Number of surgical cases: 38} \\
\hline LobeFrontal = Yes & 100 & 44.03 \\
\hline LobeParietal $=$ No & 100 & 71.07 \\
\hline LobeTemporal $=$ No & 92.11 & 59.12 \\
\hline PatientPosition $=$ Dorsal decubitus & 100 & 55.35 \\
\hline SkinIncisionForm = Arciform & 84.21 & 42.14 \\
\hline
\end{tabular}


Jannin P, Morandi X. Surgical models for computer-assisted neurosurgery. Neuroimage. 2007 Sep 1;37(3):783-91

\begin{tabular}{|l|c|c|}
\hline TargetRef = Benign tumor & 100 & 23.27 \\
\hline TargetShape = Regular & 96 & 64.15 \\
\hline TransgyralApproach = None & 88 & 63.52 \\
\hline NumberOfSteps = 6 Cluster 6 - Number of surgical cases: 17 & 45.28 \\
\hline TargetAmplitude = Cortical & 100 & 11.32 \\
\hline TargetShape = Regular & 94.12 & 64.15 \\
\hline TransgyralApproach = None & 100 & 63.52 \\
\hline NumberOfSteps = 6 & 76.47 & 45.28 \\
\hline
\end{tabular}

Table 1: Classification of 159 surgical cases with brain tumor according to parameters related to pathology description. Identification of relevant parameters with corresponding occurrences in each cluster. The number of cases per cluster is given in the black rows. For each cluster, the first rows give information about values of pathology-related parameters and the grey rows give certain parameters related to the surgical procedure and considered relevant for the cluster. The second column gives the percentage of surgical cases from the cluster verifying the corresponding property; the third column gives the percentage of surgical cases from the whole set verifying the corresponding property. 